\title{
In vivo monitoring of dihydroartemisinin- piperaquine sensitivity in Plasmodium falciparum along the China-Myanmar border of Yunnan Province, China from 2007 to 2013
}

Hui Liu', Heng-lin Yang ${ }^{1 *}$, Lin-hua Tang ${ }^{2}$, Xing-liang Li ${ }^{1}$, Fang Huang ${ }^{2}$, Jia-zhi Wang ${ }^{3}$, Chun-fu Li ${ }^{1}$, Heng-ye Wang ${ }^{1}$, Ren-hua Nie', Xiang-rui Guo ${ }^{4}$, Ying-Xue Lin ${ }^{4}$, Mei Li ${ }^{2}$, Jian Wang ${ }^{1}$ and Jian-wei Xu ${ }^{1 *}$

\begin{abstract}
Background: Artemisinin-based combination therapy (ACT) is the recommended first-line treatment of falciparum malaria in all endemic countries. Artemisinin resistance in Plasmodium falciparum has been confirmed in the Greater Mekong subregion (GMS). Dihydroartemisinin-piperaquine (DAPQ) is the most commonly used ACT in China. To understand the DAPQ sensitivity of P. falciparum, DAPQ resistance was monitored in vivo along the China-Myanmar border from 2007 to 2013.

Methods: Eligible patients with mono-infections of $P$. falciparum were recruited to this study after obtaining full informed consent. DAPQ tablets for different categories of $\mathrm{kg}$ body weight ranges were given once a day for three days. Patients were followed up for 42 days. Polymerase chain reaction (PCR) was conducted to distinguish between re-infection and recrudescence, to confirm the Plasmodium species. The data were entered and analysed by the Kaplan-Meier method. Treatment outcome was assessed according to the WHO recommended standards.

Results: 243 patients were completed valid follow-up. The fever clearance time (FCT) and asexual parasite clearance times (APCT) were, respectively, $36.5 \pm 10.9$ and $43.5 \pm 11.8$ hours, and there was an increasing trend of both FCT $(F=268.41, P<0.0001)$ and APCT $(F=88.6, P<0.0001)$ from 2007 to 2013. Eight $3.3 \%, 95 \%$ confidence interval, 1.4-6.4\%) patients present parasitaemia on day three after medication; however they were spontaneous cure on day four. 241 (99.2\%; 95\% Cl, 97.1-99.9\%) of the patients were adequate clinical and parasitological response (ACPR) and the proportions of ACPR had not changed significantly from 2007 to $2013\left(X^{2}=2.81, P=0.7288\right)$.

Conclusion: In terms of efficacy, DAPQ is still an effective treatment for falciparum malaria. DAPQ sensitivity in P. falciparum had not significantly changed along the China-Myanmar border of Yunnan Province, China. However more attentions should be given to becoming slower fever and parasite clearance.
\end{abstract}

Keywords: Plasmodium falciparum, Dihydroartemisinin-piperaquine, In vivo test, Resistance, China-Myanmar border

\footnotetext{
*Correspondence: yang_henglin@sohu.com; xjw426@163.com

YYunnan Institute of Parasitic Diseases, Yunnan Provincial Center of Malaria

Research, Yunnan Provincial Collaborative Innovation Center for Public

Health and Disease Prevention and Control, Yunnan Provincial Key

Laboratory of Vector-borne Diseases Control and Research, Puer 665000,

China

Full list of author information is available at the end of the article
} 


\section{Background}

Malaria remains one of the major global public health problems [1]. Intensive efforts in controlling malaria are producing impressive results. The number of malaria cases has fallen by more than half in $40 \%$ of malaria endemic countries in the recent decade. Estimates suggest that nearly 750,000 lives have been saved in Africa alone thanks to malaria control measures [2]. Artemisininbased combination therapy is the recommended firstline treatment of falciparum malaria in all endemic countries. However, the emergence of artemisinin resistance in malaria parasites is threatening malaria control and elimination programmes. Artemisinin resistance in Plasmodium falciparum has been identified and confirmed in Cambodia, Thailand, Myanmar, and Vietnam [2-10]. Other suspected foci have been identified in the Greater Mekong Subregion (GMS), but are not yet confirmed. The threat must be taken seriously. Resistance to previous generations of anti-malarials spread rapidly around the world, resulting in an increase in child mortality and untold number of deaths [2]. The artemisininbased combination therapy (ACT) is the most potent weapon in treating falciparum malaria [11] and no other anti-malarial is available that offers the same level of efficacy and tolerability. In order to contain artemisinin resistance, the World Health Organization (WHO) Global Malaria Programme launched the Global Plan for Artemisinin Resistance Containment. Surveillance of artemisinin sensitivity in $P$. falciparum is one of important components to prevent the emergence of new foci of resistance, as well as to limit the spread of resistance [2].

China has declared a national policy for malaria elimination by $2020[12,13]$. ACT is the first-line therapy for falciparum malaria and dihydroartemisinin-piperaquine (DAPQ) is the most common anti-malarial drug in China [14]. Historically, population movement has contributed to the spread of disease. Failure to consider this factor contributed to failure in malaria eradication campaigns in the 1950s and 1960s [15,16]. Resistance to anti-malarial drugs has often threatened malaria elimination efforts and historically has led to the short-term resurgence of malaria incidences and deaths [17]. With the development of modern transportation, the world is becoming smaller and smaller. China has the largest population in the world and Chinese people move around globally [18], especially to some parts of the GMS where artemisinin resistance has been identified and confirmed. The Yunnan Province is in southwestern China and belongs to the GMS. The Chinese Myanmar border is one of the very few remaining areas of malaria transmission in all of China. In 2013, only 423 malaria cases, 81 (19.1\%) $P$. falciparum, were reported along the border in China, and the annual parasite incidence was only 0.9 (95\% CI, 0.8-1.0) per ten thousand. Monitoring artemisinin resistance in Yunnan Province would contribute both to malaria elimination in China and in containing global artemisinin resistance. From 2007 to 2013, DAPQ resistance was monitored in vivo to determine the dynamics of $P$. falciparum sensitivity to DAPQ on the China- Myanmar border.

\section{Methods}

\section{Surveillance sites and time}

China and Myanmar share 2,185 kilometre border. There are 19 counties of China and fives special regions of Myanmar on the border. Populations includes 4,687, 896 residents of 19 counties of Yunnan Province of China and 586,000 residents of the five Special Regions of Myanmar. Due to the complex emergency situation in the five Special Regions of Myanmar, where fell outside the coverage of national malaria control efforts supported by Myanmar's Ministry of Health, effective access of malaria interventions could only be achieved from China. In March, 2008, the baseline survey of evaluation indicator for the sixth grant to China of the Global Fund to fight AIDS, Tuberculosis and Malaria showed that the parasite prevalence rate was $13.6 \%$ (761/5585, 95\% CI: 12.7-14.6\%), and the proportion of P. falciparum among slides with parasites was 38.1\% (290/761, 95\% CI: 34.641.7\%) in the five Special Regions of Myanmar. In eliminating settings, malaria predominantly occurs in border areas and imported cases tend to represent a majority of recorded cases [19]. In order to recruit malaria patients for the study, surveillance was conducted in Yingjiang of Dehong Prefecture in 2007, Menglian of Puer Prefecture in 2009, in both Tengchong of Baoshan Prefecture and Yingjiang of Dehong Prefecture from 2010 to 2013 (Figure 1).

\section{Patients and recruiting criteria}

Patients whose axillary temperature was $\geq 37.5^{\circ} \mathrm{C}$ or with a history of fever during the previous 24 hours were diagnosed by using microscopy of thick and thin blood smears. Patients with mono-infections of $P$. falciparum were recruited to this study, after obtaining full informed consent. Only patients older than one year, body weight $\geq 5 \mathrm{~kg}$, presenting with parasite density 500100,000 parasites per $\mu \mathrm{l}$ were enrolled into the study. Imported malaria was identified as patients who had travelled from endemic areas of Myanmar within one month and were diagnosed as malaria in China [13]. Patients were excluded from the study if any of the following criteria were present: (1) positive pregnancy test or breastfeeding; (2) complicated malaria; (3) having taken any anti-malarial, tetracycline and sulphonamides derivatives drugs within the past seven days; (4) history of hypersensitivity to any of the study drugs; (5) presence of febrile conditions due to diseases other than malaria (e.g. measles, acute lower respiratory tract 


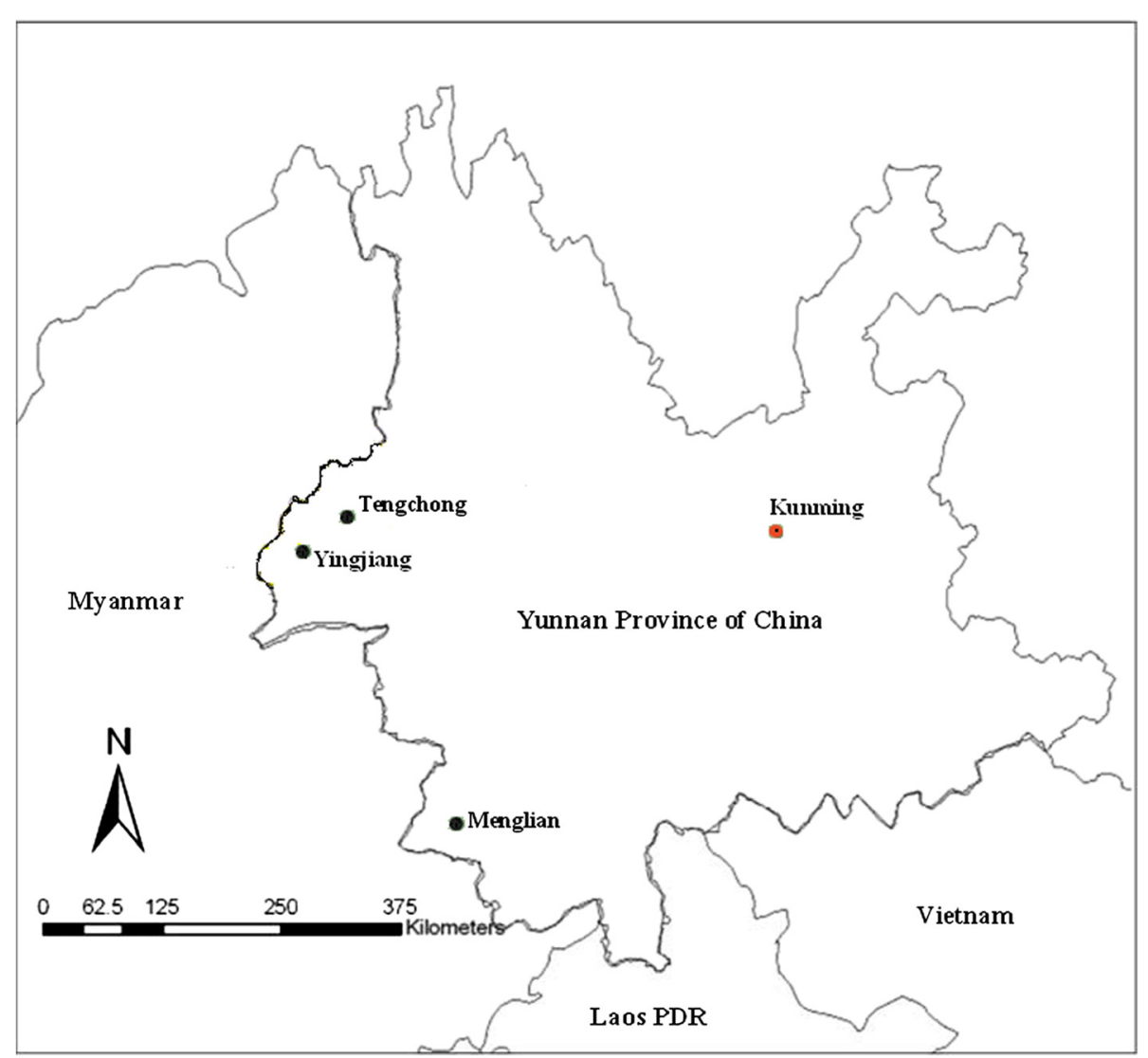

Figure 1 Surveillance sites: Menglian, Tengchong and Yingjian in Yunnan Province of China and relative to neighbouring countries.

infection, severe diarrhoea with dehydration) or other known underlying chronic or severe diseases (e.g. cardiac, renal and hepatic diseases, HIV/AIDS); (6) over 60 years; (7) presence of severe malnutrition (defined as a child whose growth standard is below $-3 \mathrm{z}$-score, has symmetrical oedema involving at least the feet or has a mid-upper arm circumference $<110 \mathrm{~mm}$ ); and, (8) unable to follow-up [20,21].

\section{Drug and administration}

DAPQ was manufactured by Zhejiang Holley Nanhu Pharmaceutical Co. Ltd, and provided by the West Pacific Office of WHO (WPRO/WHO). The drug quality was controlled by WPRO/WHO too. The batch numbers of DAPQ were 600807 (manufactured on 30 Aug 2007), 460909 (23 Sep 2009) and 400911 (20 Sep 2011). Each tablet contains $40 \mathrm{mg}$ base dihydroartemisinin and $320 \mathrm{mg}$ piperaquine phosphate. DAPQ was given once a day for three days and the dosing were based on the recommendation of WPRO/WHO. For convenient administration, the doses were calculated into tablets for different categories of $\mathrm{kg}$ body weight ranges (Table 1). The treatment intake was observed while the patients visited the hospital as requested for the first three days. Patients of late clinical failure and late parasitological failure were subsequently given a total dose of artemisininnaphthoquine $24.5 \mathrm{mg} / \mathrm{kg}$ (naphthoquine $7 \mathrm{mg} / \mathrm{kg}$ and artemisinin $17.5 \mathrm{mg} / \mathrm{kg}$ ), once a day for three days [22].

\section{Laboratory and in vivo clinical monitoring}

Parasite microscopy was conducted and axillary temperatures were measured on admission and every 8-12 hours in the first three days. Patients were asked to visit the hospital and further parasitological examinations were performed on day 7, 14, 21, 28, 35 and 42. In a case of patient had not visited the hospital, a researcher actively visited the patient home to prepare blood smears. Malaria blood

Table 1 Number of DAPQ tablets for each category of body weight range

\begin{tabular}{lllll}
\hline $\begin{array}{l}\text { Body weight } \\
\text { range }(\mathbf{k g})\end{array}$ & $\begin{array}{l}\text { No. tablets, } \\
\text { Day 1 }\end{array}$ & $\begin{array}{l}\text { No. tablets, } \\
\text { Day 2 }\end{array}$ & $\begin{array}{l}\text { No. tablets, } \\
\text { Day 3 }\end{array}$ & $\begin{array}{l}\text { Total } \\
\text { Tablets }\end{array}$ \\
\hline $5-10$ & $1 / 2$ & $1 / 2$ & $1 / 2$ & $1 \frac{1}{2}$ \\
$10.1-19$ & 1 & 1 & 1 & 3 \\
$19.1-30$ & $11 / 2$ & $11 / 2$ & $11 / 2$ & $4 \frac{1}{2}$ \\
$30.1-40$ & 2 & 2 & 2 & 6 \\
$>40$ & 3 & 3 & 3 & 9 \\
\hline
\end{tabular}


films were stained with Giemsa, and slides were examined by two independent microscopists and considered negative if no parasites were seen after examination of 200 oilimmersion fields in a thick blood film. Parasite clearance was defined as no asexual parasite per 500 white blood cells being detected in two continuously microscopic examinations with an 8-12 hr interval. Fever clearance was defined as axillary temperatures $<37.1^{\circ} \mathrm{C}$ in duration of 24 hours. Parasites were counted per 500 white blood cells. The number of parasites was calculated as per $\mu \mathrm{l}$ of blood by the level of 8,000 of leukocyte per $\mu \mathrm{l}$ [23]. A filter paper dried blood spot (about 100ul blood) was prepared on admission, day $7,14,21,28,35$ and 42 , day of treatment failure or at any other unscheduled visit, and subsequently stored in plastic zip bags containing silica gel dessicant. Polymerase chain reaction (PCR) was performed respectively to distinguish between reinfection and recurrence of asexual parasites of blood stages, to confirm the Plasmodium species or to detect mixed infection. DNA extraction and genotype analysis were conducted based on investigation of the three polymorphic genetic markers $m s p 1, m s p 2$, and glurp, according to WHO recommended procedures [24]. Recrudescence was defined as at least one identical allele for each of the three markers in the pre-treatment and post-treatment samples. New infections were diagnosed when all alleles for at least one of the markers differed between the two samples. Cases with new infection were excluded from the analysis [25]. In case of failure after day 7 , patients whose PCR results were unknown were excluded from the analysis too [11].

\section{Classification standards for treatment outcome}

Treatment outcome was categorized based on the WHO definitions for early treatment failure (ETF), late clinical failure (LCF), late parasitological failure (LPF), and adequate clinical and parasitological response (ACPR). The ETF definition was to conform to any one of the criteria: (1) danger signs or severe malaria on day 1, 2 or 3 , in the presence of parasitaemia; (2) parasitaemia on day 2 higher than on day 0 , irrespective of axillary temperature; (3) parasitaemia on day 3 with axillary temperature $\geq 37.5^{\circ} \mathrm{C}$; and, (4) parasitaemia on day $3 \geq 25 \%$ of count on day 0 . The LCF definition was to satisfy any one of the criteria: (1) danger signs or severe malaria in the presence of parasitaemia on any day between day 4 and day 42 in patients who did not previously meet any of the criteria of ETF; and, (2) presence of parasitaemia on any day between day 4 and day 42 with axillary temperature $\geq 37.5^{\circ} \mathrm{C}$ in patients who did not previously meet any of the criteria of ETF. LPF definition is to satisfy presence of parasitaemia on any day between day 7 and day 42 with axillary temperature $<37.5^{\circ} \mathrm{C}$ in patients who did not previously meet any criteria of ETF or LCF. ACPR definition was to satisfy absence of parasitaemia on day 42 , irrespective of axillary temperature, in patients who did not previously meet any criteria of ETF, LCF or LPF [20,21].

\section{Statistical analysis and resistance assessment}

The data were by double independent data entry and analysed by the Kaplan-Meier method [22,26]. The patients of loss to follow-up and withdrawal from the study were not involved into the analysis. Mean fever and parasite clearance time were compared by covariance through Epi Info 6.04 [22,26]. The proportions of ETF, LCF, LPF and ACPR were, respectively, compared by Chi-square test for trend of quantitative data [27]. The treatment outcome was assessed on the basis of parasite clearance from the blood.

\section{Ethical approval}

According to the Helsinki Declaration, ethical approval for the study was granted by the Ethics Committee of Yunnan Institute of Parasitic Diseases, China. The purpose of the study was explained and then approval was sought from patients and their caretakers. Informed written consent was obtained from patient or carers of Child patients. All results were kept confidential and were unlinked to any identifying information.

\section{Results}

A total of 290 falciparum malaria patients were recruited in the study during 2007-2013 (the study was interrupted in 2008 because of shortage of funding), 22(7.6\%) withdrew, 25(8.6\%) were lost at follow-up, and 243 (83.8\%) completed valid follow-up, of which 178 (72.3\%) were Burmese (Table 2). The fever clearance time (FCT) and asexual parasite clearance times (APCT) were, respectively, $36.5 \pm 10.9$ and $43.5 \pm 11.8$ hours. The results of covariance analysis showed an increasing trend of both FCT $(\mathrm{F}=268.41, \mathrm{P}<0.0001)$ and APCT $(\mathrm{F}=88.6$, $\mathrm{P}<0.0001)$ (Table 3$)$. Eight $(3.3 \%, 95 \%$ confidence interval, 1.4-6.4\%) patients present parasitaemia on day 3 after medication, three in 2010, one in 2011, three in 2012 and one in 2013 (Figure 2); and then parasites were spontaneously cleared by day 4 . The results showed that 241 (99.2\%; 95\% CI, 97.1-99.9\%) of the patients were ACPR, one ETF in 2012 and one LCF in 2007, without LPF (Table 3). The results of Chi-square test for trend of quantitative data showed that percentages of ACPR had not changed significantly from 2007 to $2013\left(X^{2}=2.81\right.$, $\mathrm{P}=0.7288$ ) (Table 3 ). The ETF patient was cured spontaneously by day four. The LCF patients responded well to the three-day treatment regimen of artemisininnaphthoquine tablets. The ETF and LCF cases were imported from the neighbouring districts of Myanmar. The PCR identified the LCF as recrudescence on day 28 in 2007, and did not identify any new infection of P. falciparum. 
Table 2 Baseline characteristics of falciparum malaria patients on the China-Myanmar border, Yunnan Province, China

\begin{tabular}{|c|c|c|c|c|c|c|c|}
\hline & $2007(n=38)$ & $2009(n=71)$ & $2010(n=27)$ & $2011(n=17)$ & $2012(n=63)$ & $2013(n=27)$ & Total $(n=243)$ \\
\hline \multicolumn{8}{|l|}{ Sex } \\
\hline Male (\%) & $19(50)$ & $32(45.1)$ & $21(77.8)$ & $17(100)$ & $44(69.8)$ & $20(74.1)$ & $153(63.0)$ \\
\hline Female (\%) & $19(50)$ & $39(54.9)$ & $6(22.2)$ & 0 & $19(30.2)$ & $7(25.9)$ & $90(37.0)$ \\
\hline \multicolumn{8}{|l|}{ Nationality } \\
\hline Chinese (\%) & $1(2.6)$ & $10(14.1)$ & $3(11.1)$ & $17(100)$ & $21(33.3)$ & $13(48.1)$ & $65(26.7)$ \\
\hline Burmese (\%) & $37(97.4)$ & $61(85.9)$ & $24(88.9)$ & 0 & $42(66.7)$ & $14(51.9)$ & $178(72.3)$ \\
\hline \multicolumn{8}{|l|}{ Age (years) } \\
\hline Mean $( \pm S D)$ & $16.3(9.2)$ & $25.0(3.8)$ & $26.3(4.2)$ & $33.6(1.2)$ & $26.1(8.3)$ & $28.0(9.6)$ & $25.9(6.8)$ \\
\hline Range & $2-60$ & $7-56$ & $2-49$ & $19-48$ & $4-60$ & $2-56$ & $2-60$ \\
\hline \multicolumn{8}{|c|}{ Body temperature $\left({ }^{\circ} \mathrm{C}\right)$} \\
\hline Mean $( \pm S D)$ & $38.3(0.7)$ & $38.5(0.6)$ & $38.3(0.5)$ & $39.3(0.3)$ & $38.8(0.9)$ & $38.7(0.8)$ & $38.6(0.7)$ \\
\hline Rang & $37-40.5$ & $37.2-39.4$ & $36.8-40.4$ & $38.0-40.0$ & $36.1-40.4$ & $37.8-39.7$ & $36.1-39.7$ \\
\hline \multicolumn{8}{|c|}{ Parasite count (per ul) } \\
\hline Geometric mean & 11100 & 11257 & 73784 & 38776 & 24257 & 24886 & 30678 \\
\hline Range (per $\mu \mathrm{l}$ ) & $760-37120$ & $500-45200$ & $540-821700$ & 5129-119657 & 596-167132 & 524-162960 & $596-821700$ \\
\hline
\end{tabular}

\section{Discussion}

Plasmodium falciparum resistance to artemisinin derivatives in Southeast Asia threatens malaria control and elimination activities worldwide [28,29]. The results of the study showed that the FCT and APCT were increasing from 2007 to 2013 (Table 3); 3.3\% patients present parasitaemia on day 3 after medication, but were spontaneously cured by day 4 without treatment. The principal pharmacodynamic advantage of using artemisinins is that they accelerate parasite clearance by clearing young, circulating, ring-stage parasites and preventing the further maturation and sequestration of these parasites. This effect accounts for the rapidity of the therapeutic response, its lifesaving benefit in patients with severe malaria, and the notable gametocytocidal activity of the drugs [3,28-30]. The reduced susceptibility of ring-stage parasites causes the slow parasite clearance [29-33]. The markedly prolonged time to parasite clearance showed a decline in the efficacy of DAPQ during the six years. However, DAPQ was still highly efficacious for the treatment of falciparum malaria on the China-Myanmar border areas, presumably because of the efficacy of piperaquine

Table 3 Treatment responses of falciparum malaria patients on the China-Myanmar border, Yunnan Province, China

\begin{tabular}{|c|c|c|c|c|c|c|c|c|c|}
\hline & $2007(n=38)$ & $\begin{array}{l}2009 \\
(n=71)\end{array}$ & $\begin{array}{l}2010 \\
(n=27)\end{array}$ & $\begin{array}{l}2011 \\
(n=17) \\
\end{array}$ & $\begin{array}{l}2012 \\
(n=63)\end{array}$ & $\begin{array}{l}2013 \\
(n=27)\end{array}$ & $\begin{array}{l}\text { Total } \\
(n=243)\end{array}$ & Statistics & P-value \\
\hline \multicolumn{10}{|l|}{$\mathrm{FCT}(\mathrm{hr})$} \\
\hline Mean ( \pm SD) & $24.5(8.1)$ & $34.8(10.4)$ & $36.4(9.9)$ & $35.3(9.5)$ & $43.3(11.4)$ & $45.7(11.6)$ & $36.5(10.9)$ & $F=268.4$ & $<0.0001$ \\
\hline Range & $16-40$ & $20-48$ & $20-72$ & $22-96$ & 24-96 & $23-96$ & $16-96$ & & \\
\hline \multicolumn{10}{|l|}{$50 \%$ АРCT (hr) } \\
\hline Mean ( \pm SD) & $24.2(7.8)$ & $24.6(8.7)$ & $25.8(7.4)$ & $26.8(8.5)$ & $25.9(8.2)$ & $26.3(8.4)$ & $25.2(8.2)$ & $F=4.14$ & 0.0013 \\
\hline Range & $3-29$ & $7-25$ & $8-48$ & $8-48$ & $6-48$ & $7-48$ & $3-48$ & & \\
\hline \multicolumn{10}{|l|}{ APCT (hr) } \\
\hline Mean ( \pm SD) & $38.4(7.5)$ & $39.8(7.9)$ & $41.8(8.4)$ & $40.9(9.2)$ & $42.6(11.3)$ & $52.6(12.3)$ & $43.5(11.8)$ & $F=88.6$ & $<0.0001$ \\
\hline Range & $19-46$ & $16-72$ & 19-72 & 18-96 & $16-96$ & $20-72$ & $16-96$ & & \\
\hline ETF $(\%, 95 \% \mathrm{Cl})$ & $0(0,0-9.3)$ & $0(0,0-7.0)$ & $0(0,0-12.8)$ & $0(0,0-19.5)$ & $1(1.6,0.04-8.5)$ & $0(0,0-12.8)$ & $1(0.4,0-2.3)$ & $x^{2}=2.61$ & 0.7591 \\
\hline LCF $(\%, 95 \% \mathrm{Cl})$ & $1(2.6,0.1-13.8)$ & $0(0,0-5.1)$ & $0(0,0-12.8)$ & $0(0,0-19.5)$ & $0(0,0-5.7)$ & $0(0,0-12.8)$ & $1(0.4,0-2.3)$ & $X^{2}=5.00$ & 0.4164 \\
\hline LPF $(\%, 95 \% C l)$ & $0(0,0-9.3)$ & $0(0,0-5.1)$ & $0(0,0-12.8)$ & $0(0,0-19.5)$ & $0(0,0-5.7)$ & $0(0,0-12.8)$ & $0(0,0-1.5)$ & - & - \\
\hline ACPR $(\%, 95 \% \mathrm{Cl})$ & $\begin{array}{l}37 \text { (97.4, } \\
82.7-99.4)\end{array}$ & $\begin{array}{l}71(100 \\
94.9-100)\end{array}$ & $\begin{array}{l}27(100 \\
87.2-100)\end{array}$ & $\begin{array}{l}17(100 \\
80.5-100)\end{array}$ & $\begin{array}{l}62 \text { (98.4, } \\
91.5-99.9)\end{array}$ & $\begin{array}{l}27(100 \\
87.2-100)\end{array}$ & $\begin{array}{l}241 \text { (99.2, } \\
97.1-99.9)\end{array}$ & $X^{2}=2.81$ & 0.7288 \\
\hline
\end{tabular}

Note: $\mathrm{FCT}=$ fever clearance times (hours), $\mathrm{APCT}=$ asexual parasite clearance times (hours); $\mathrm{ETF}=$ early treatment failure, $\mathrm{LCF}=$ late clinical failure, $\mathrm{LPF}=$ late parasitological failure, $A C P R=$ adequate clinical and parasitological response, and $95 \% \mathrm{Cl}=95 \%$ confidence interval. 


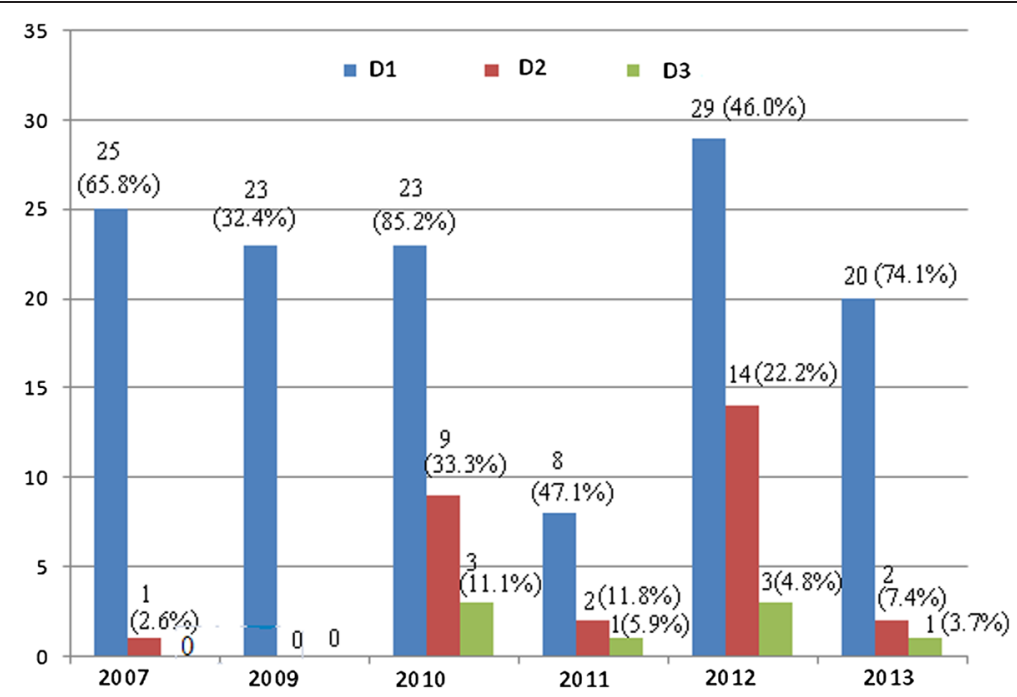

Figure 2 Number (\%) of patients of parasitaemia presence on day 1, 2 and 3 after medication.

phosphate. $99.2 \%$ of the patients were ACPR and the proportions of ACPR had not changed significantly from 2007 to $2013\left(\mathrm{X}^{2}=2.81, \mathrm{P}=0.7288\right)$.

DAPQ sensitivity of $P$. falciparum had been monitored on the China-Myanmar border. Sun et al. reported that DAPQ was efficacious for falciparum malaria treatment in 2006 [34], however Liu et al. reported a 97.0\% of cumulative success rate (CSR) and reduced DAPQ sensitivity in P. falciparum of a trial in which the only eight tablets of DAPQ (a dosage for $>40 \mathrm{~kg}$ body weight) were used for uncomplicated falciparum malaria treatment [35]. In the study by Liu et al., the technical limitation was to not use PCR to distinguish between re-infection and recrudescence, and they conduct their study in Wa State of Myanmar, where $P$ falciparum prevalence was high during 2007-2008. The technical limitation and the different regimen might result in the declined CSR, and geographic differences might be one of explanations for reported differences too. Huang et al. reported a 95.9\% (47/49) of artesunate CSR and a high frequency of mutations in pfcrt, pfdhfr and pfdhps associated with chloroquine and sulphadoxine-pyrimethamine resistance and no pfatp6 mutation in P. falciparum [36]. Wang et al. reported low levels of point mutations in pfmdr1 and pfatp 6 prevalence and no pfmdr 1 gene amplification detected [37]. The results of the two molecular epidemiology studies showed lack of molecular basis of artemisinin resistance along China-Myanmar border. All these investigations together were not enough to confirm the artemisinin resistance in $P$. falciparum along the China-Myanmar border.

Several limitations should be considered while using the results of study. Firstly, both total and yearly sample size were limited by the difficulty in recruiting patients because of low malaria incidence in eliminating settings, only total 1011 falciparum malaria cases were reported during 2010-2013. Secondly, the surveillance activity was interrupted in 2008 because of shortage of funding. Thirdly, the surveillance sites had to be changed in order to recruit falciparum malaria patients, in Yingjiang in 2007, Menglian in 2009, in both Tengchong and Yingjiang from 2010 to 2013, however all the three sites are on the China-Myanmar border. Fourthly, the study did not use any pharmacokinetics (PK) measurement to ascertain drug absorption and to characterize the concentration-time profile of drugs and the relevant covariates (e.g. immunity, young age and drug interactions), so it did not exclude confounding effect of some variables that could influence APCT [38]. Fifthly, mutations of PF3D7_1343700 kelch propeller domain (K13-propeller) are important determinants of artemisinin resistance [29]; the study did not investigate mutant K13-propeller alleles. According to the new working definition of ACT or artesunate monotherapy resistance, the suspected resistance is as evidenced by either $\geq 5 \% \mathrm{~K} 13$ propeller domain mutants or $\geq 10 \%$ of patients who are parasitaemic on day three; and the confirmed resistance should satisfy both $\geq 5 \%$ K13-propeller domain mutants and $\geq 10 \%$ of patients with parasitaemia on day three, or by the persistence of parasites for seven days, or by the presence of parasites on day three and recrudescence within $28 / 42$ days $[29,39]$. The monitored results showed that only $3.3 \%(8 / 243)$ of patients presented parasitaemia by day three and only $0.8 \%(2 / 243)$ were ETF or LCF. According to the new working definition DAPQ was still sensitive in P. falciparum along the China-Myanmar border.

While artemisinin resistance was found on the western border of Cambodia-Thailand and the western border of Thailand-Myanmar [40], P. falciparum was still sensitive to artemisinins on the China-Myanmar border. The 
genetic diversity of malaria parasites and multiclonal infections are correlated with transmission intensity as well as the spread of anti-malarial resistance. Despite the intensified control efforts and the decline of malaria prevalence along China-Myanmar border, the parasite population size and transmission intensity remained high enough to allow effective genetic recombination of the parasites and continued maintenance of genetic diversity in ChinaMyanmar border area [41]. Therefore, surveillance for artemisinin resistance in P. falciparum should be maintained and containment activities should be further strengthened to monitor the spread of resistance, define therapeutic and operational strategies to understand its molecular basis and counter its impact [28,42]. In terms of monitoring artemisinin resistance, treatment failure of ACT is more direct and accurate [43], so it may be more useful in artemisinin sensitivity surveillance.

Detection of drug efficacy is only the first step to producing accessible and useful information for decision makers. The translation of increased access to data on health outcomes into usable evidence for rational policy and planning requires a global coordination and communication effort [44]. In terms of containment measures, a $97.5 \%$ of DAPQ CSR for P. falciparum treatment had been reported in Hainan Province of China in 2004 [45] and then $100 \%$ in 2008 [46], P. falciparum has been eliminated by using the strategy of early diagnosis and treatment (EDT) with ACT [47]. The emergence of resistance to artesunate in $P$. falciparum, the strategy of EDT with ACT has reduced malaria in the migrant population living on the Thai-Myanmar border [48]. The addition of one dose of primaquine to ACT could help to counter the spread of artemisinin resistance [49]. Despite the 8-aminoquinoline compound cause patients with G6PD deficiency haemolysis, it can sterilize gametocytes of $P$. falciparum. Lower doses of the gametocytocide would be safer, might still be very effective for blocking transmission.

\section{Conclusion}

In terms of efficacy, DAPQ is still effective for falciparum malaria treatment. DAPQ sensitivity in P. falciparum had not significantly changed along the China-Myanmar border of Yunnan Province, China. However more attentions should be given to becoming slower fever and parasite clearance, surveillance for artemisinin resistance in P. falciparum should be maintained and containment measures are urgently needed.

\section{Competing interests}

The authors declare that they have no competing interests.

\section{Authors' contributions}

$L-H T, H-L Y, H L, F H$ and $M L$ designed the study and developed the protocol. $J-W X$ and $\mathrm{HL}$ analysed and interpreted the data. HL organized and supervised the study in field. L-HT, H-LY, FH and ML monitored the study in general. HL,
$X-L L, J-Z W, C-F L, H-Y W, R-H N, X-R G, Y-X L$ and JW conducted the study in field, and entered the data. FH and $M L$ performed the PCR test. J-WX and $\mathrm{HL}$ wrote the first draft of the paper. All authors read and approved the final manuscript.

\section{Acknowledgements}

This study was supported by The WHO Mekong Malaria Programme (WP/10/ MVP/005837), the sixth (CHN-607-G09-M) and tenth (CHN-011-G15-M) grant to China of the Global Fund to fight AIDS, Tuberculosis and Malaria (GFATM). We thank all participants for their contribution of time and patience in the study. We also thank staff of Yunnan Institute of Parasitic Diseases (YIPD) for logistic support, and clinical and laboratory staff of Menglian, Tengchong and Yangjiang County Center for Disease Control and Prevention for their hard work. The opinions expressed are those of the authors and do not necessarily reflect those of WHO, GFATM and YIPD.

\section{Author details}

${ }^{1}$ Yunnan Institute of Parasitic Diseases, Yunnan Provincial Center of Malaria Research, Yunnan Provincial Collaborative Innovation Center for Public Health and Disease Prevention and Control, Yunnan Provincial Key Laboratory of Vector-borne Diseases Control and Research, Puer 665000 China. ${ }^{2}$ National Institute of Parasitic Diseases, Chinese Center for Disease Control and Prevention, Shanghai 200025, PR China. ${ }^{3}$ Tengchong County Center for Disease Control and Prevention, Tengchong 679100, China. ${ }^{4}$ Yangjiang County Center for Disease Control and Prevention, Yingjiang 679300, China.

Received: 7 November 2014 Accepted: 25 January 2015

Published online: 05 February 2015

\section{References}

1. WHO. World malaria report 2012. Geneva: World Health Organization; 2012

2. WHO. The global plan for artemisinin resistance containment. Geneva: World Health Organization; 2011.

3. Witkowski B, Amaratunga C, Khim N, Sreng S, Chim P, Kim S, et al. Novel phenotypic assays detect artemisinin-resistant Plasmodium falciparum malaria in Cambodia: in-vitro and ex-vivo drug response studies. Lancet Infect Dis. 2013;13:1043-9.

4. Noedl H, Se Y, Schaecher K, Smith B, Socheat D, Fukuda M. Evidence of artemisinin-resistant malaria in western Cambodia. N Engl J Med. 2008:359:2619-20.

5. Amaratunga C, Sreng S, Suon S, Phelps E, Stepniewska K, Lim P, et al. Artemisinin-resistant Plasmodium falciparum in Pursat province, western Cambodia: a parasite clearance rate study. Lancet Infect Dis. 2012;12:851-8.

6. Amaratunga $C$, Mao S, Sreng S. Slow parasite clearance rates in response to artemether in patients with severe malaria. Lancet Infect Dis. 2013;13:113-4.

7. Hien TT, Thuy-Nhien NT, Phu NH. In vivo susceptibility of Plasmodium falciparum to artesunate in Binh Phuoc Province, Vietnam. Malar J. 2012;11:355.

8. Phyo AP, Nkhoma S, Stepniewska K, Ashley E, Nair S, Gready R, et al. Emergence of artemisinin-resistant malaria on the western border of Thailand: a longitudinal study. Lancet. 2012;379:1960-6.

9. Bangchang K, Karbwang J. Emerging artemisinin resistance in the border areas of Thailand. Clin Pharmacol. 2013:6:307-22.

10. Kyaw MP, Nyunt MH, Chit K. Reduced susceptibility of Plasmodium falciparum to artesunate in southern Myanmar. PLoS One. 2013:8:e57689.

11. WHO. Guidelines for the treatment of malaria. Geneva: World Health Organization; 2006. p. 1-66.

12. $\mathrm{MOH}$ of People's Republic of China. From malaria control to elimination: a revised national malaria strategy 2010-2015. Beijing: Ministry of Health; 2009. p. 2-4.

13. $\mathrm{MOH}$ of People's Republic of China. Malaria elimination action plan 2010-2020. Beijing: Ministry of Health; 2010. p. 1-2 [in Chinese].

14. $\mathrm{MOH}$ of People's Republic of China. Malaria control manual. Beijing: Ministry of Health; 2007. p. 33-43 [in Chinese].

15. Martens P, Hall L. Malaria on the move: human population movement and malaria transmission. Emerg Infect Dis. 2000:6:103-9.

16. $\mathrm{Xu} \mathrm{JW}$, Liu H. The challenges of malaria elimination in yunnan province, people's republic of China. Southeast Asian J Trop Med Public Health. 2012;43:819-24. 
17. Petersen I, Eastman R, Lanzer M. Drug-resistant malaria: molecular mechanisms and implications for public health. FEBS Lett. 2011;585:1551-62.

18. Bräutigam D. Local entrepreneurship in Southeast Asia and Subsaharan Africa: networks and linkages to the global economy. Washington, DC: School of International Service, American University; 1998.

19. Cotter C, Sturrock HJW, Hsiang MS, Liu J, Phillips AA, Hwang J, et al. The changing epidemiology of malaria elimination: new strategies for new challenges. Lancet. 2013;382:900-11.

20. WHO. Assessment and monitoring of antimalarial drug efficacy for the treatment of uncomplicated falciparum malaria. Geneva: World Health Organization; 2003. (WHO/RBM/HTM/2003.50) http://www.who.int/malaria/ resistance.

21. WHO. Method for surveillance of antimalarial drug efficacy. Geneva: World Health Organization; 2009. p. 12-82.

22. Liu H, Yang HL, Xu JW, Wang JZ, Nie RH, Li CF. Artemisinin-naphthoquine combination versus chloroquine-primaquine to treat vivax malaria: an open-label randomized and non-inferiority trial in Yunnan Province, China. Malar J. 2013;12:409.

23. Yang HL, Yang $Y$, Yang PF. Monitoring Plasmodium falciparum chloroquine resistance in Yunnan Province, China, 1981-2006. Acta Trop. 2008;108:44-9.

24. WHO. Methods and techniques for clinical trials on antimalarial efficacy: genotyping to identify parasite populations. Geneva: World Health Organization; 2008

25. Nhama A, Bassat Q, Enosse S, Nhacolo A, Mutemba R, Carvalho E, et al. In vivo efficacy of artemether-lumefantrine and artesunate-amodiaquine for the treatment of uncomplicated falciparum malaria in children: a multisite, open-label, two-cohort, clinical trial in Mozambique. Malar J. 2014;13:309.

26. Stepniewska K, White NJ. Some considerations in the design and interpretation of antimalarial drug trials in uncomplicated falciparum malaria. Malar J. 2006:5:127

27. Xu JW, Liao YM, Liu H, Nie RH, Havumaki J. Use of bed nets and factors that influence bed net use among Jinuo ethnic minority in Southern China. PLoS One. 2014;9:e103780. doi:10.1371/journal.pone.0103780.

28. Dondorp A, Nosten F, Yi P, Das D, Phyo AP, Tarning J, et al. Artemisinin resistance in Plasmodium falciparum malaria. N Engl J Med. 2009;361:455-67.

29. Ariey F, Witkowski B, Amaratunga C, Beghain J, Langlois AC, Khim N, et al. A molecular marker of artemisinin-resistant Plasmodium falciparum malaria. Nature. 2014;505:50-5.

30. White NJ. Qinghaosu (artemisinin): the price of success. Science 2008;320:330-4.

31. Witkowski B, Khim N, Chim P, Kim S, Ke S, Kloeug N, et al. Reduced artemisinin susceptibility of Plasmodium falciparum ring stages in western Cambodia. Antimicrob Agents Chemother. 2013;57:914-23.

32. Mok S, Imwong M, Mackinnon MJ, Sim J, Ramadoss R, Yi P, et al. Artemisinin resistance in Plasmodium falciparum is associated with an altered temporal pattern of transcription. BMC Genomics. 2011;12:391.

33. Chotivanich K, Tripura R, Das D, Yi P, Day N, Pukrittayakamee S. Laboratory detection of artemisinin resistant Plasmodium falciparum. Antimicrob Agents Chemother. 2014;58:3157-61.

34. Sun XD, Zhang ZX, Liu DQ. Clinical study on the efficacy of dihyroartemisinin and piperaquine in treatment of multi-drug resistant Plasmodium falciparum [in Chinese]. Chin Trop Med. 2006;6:211-2.

35. Liu H, Yang HL, Zhang J, Li CF, Nie RH, Wang HY. Clinical trial on efficacy of compound dihydroartemisinin-piperaquine for treatment of uncomplicated falciparum malaria in Myanmar [in Chinese]. Chin J Parasit Parasitic Dis. 2011;29:296-8

36. Huang F, Tang LH, Yang HL, Zhou SS, Sun XD, Liu H. Therapeutic efficacy of artesunate in the treatment of uncomplicated Plasmodium falciparum malaria and anti-malarial, drug-resistance marker polymorphisms in populations near the China-Myanmar border. Malar J. 2012;11:278.

37. Wang Z, Parker D, Meng H, Wu L, Li J, Zhao Z, et al. In vitro sensitivity of Plasmodium falciparum from China-Myanmar border area to major ACT drugs and polymorphisms in potential target genes. PLoS One. 2012:7:e30927. doi:10.1371/ journal.pone.0030927.

38. WHO. Methods and techniques for assessing exposures to antimalarial drugs in clinical field studies. Geneva: World Health Organization; 2011.

39. WHO. Status report on artemisinin resistance. Geneva: World Health Organization; 2014

40. Kyaw TT, Hlaing T, Thimasarn K, Mon KM, Galappaththy G, Plasai V, et al. Containing artemisinin resistance of Plasmodium falciparum in Myanmar:
Achievements, challenges and the way forward. Southeast Asia J Public Health. 2014;3:90-4.

41. Yuan LL, Zhao H, Lanou Wu L, Li XM, Parkerd D, Xu SH, et al. Plasmodium falciparum populations from northeastern Myanmar display high levels of genetic diversity at multiple antigenic loci. Acta Trop. 2013;125:53-9.

42. Briena CO, Henricha P, Passia N, Fidock D. Recent clinical and molecular insights into emerging artemisinin resistance in Plasmodium falciparum. Curr Opin Infect Dis. 2011:24:570-7.

43. Krishna S, Kremsner PG. Antidogmatic approaches to artemisinin resistance: reappraisal as treatment failure with artemisinin combination therapy. Trends Parasitol. 2013;29:313-7.

44. Guerin PJ, Bates SJ, Sibley CH. Global resistance surveillance: ensuring antimalarial efficacy in the future. Curr Opin Infect Dis. 2009;22:593-600.

45. Song JP, Chen PQ, Xu Y. Randomized-control trial of compound dihydroartemisinin in treating uncomplicated falciparum malaria in Hainan Province of China [in Chinese]. J Guangzhou Uni of Trad Chin Med. 2004:21:9-12.

46. Wang SQ, Christophel EM, Lin SG. Efficacy of dihydroartemisinin-piperaquine and artemether-lumefantrine in the treatment of uncomplicated falciparum malaria in Hainan [in Chinese]. Chin J Parasit Parasitic Dis. 2008;26:50-2.

47. Xia ZG, Yang MN, Zhou SS. Malaria situation in the People's Republic of China in 2011 [in Chinese]. Chin J Parasitol Parasit Dis. 2012;30:419-22.

48. Carrara VI, Lwin KM, Phyo AP, Ashley E, Wiladphaingern J, Sriprawat K, et al. Malaria burden and artemisinin resistance in the mobile and migrant population on the Thai-Myanmar border, 1999-2011: an observational study. PLoS Med. 2013;10:e1001398.

49. White N. Primaquine to prevent transmission of falciparum malaria. Lancet Infect Dis. 2013:13:175-81.

\section{Submit your next manuscript to BioMed Central and take full advantage of:}

- Convenient online submission

- Thorough peer review

- No space constraints or color figure charges

- Immediate publication on acceptance

- Inclusion in PubMed, CAS, Scopus and Google Scholar

- Research which is freely available for redistribution 